\title{
Stability of a General Class of Distributed Algorithms for Power Control in Time-Varying Wireless Networks
}

\author{
Eoin Devane and Ioannis Lestas, Member, IEEE
}

\begin{abstract}
In order for a wireless network to function effectively, the signal power of each user's transmitter must be sufficiently large to ensure a reliable uplink connection to the receiver, but not so large as to cause interference with neighboring users. We consider a general class of distributed algorithms for the control of transmitter power allocations in wireless networks with a general form of interference nonlinearity. In particular, we allow this interference to have explicit time-dependence, allowing our analysis to remain valid for network configurations that vary with time. We employ appropriately constructed Lyapunov functions to show that any bounded power distribution obtained from these algorithms is uniformly asymptotically stable. Further, we use Lyapunov-Razumikhin functions to show that, even when the system incorporates heterogeneous, time-varying delays, any solution along which the generalized system nonlinearity is bounded must also be uniformly asymptotically stable. Moreover, in both of these cases this stability is shown to be global, meaning that every power distribution has the same asymptotic behavior. These results are also used in the paper to derive timeinvariant asymptotic bounds for the trajectories when the system nonlinearities are appropriately bounded.
\end{abstract}

Index Terms-network analysis and control, decentralized control, wireless networks, delay systems.

\section{INTRODUCTION}

$\mathbf{I}$ $\mathrm{N}$ the design of a wireless network, one important factor to control is the power of the uplink signals transmitted by individual nodes to their corresponding receivers. In practice, these nodes represent the onboard transmitters of the various devices connected to the network, while each node's corresponding receiver will be one of a number of local wireless base stations as determined by the network's base station assignment rule. For the network to function effectively, each user's transmitted signal power must be sufficiently high to ensure that the node retains a reliable connection, but not so high that it might cause interference with neighboring nodes or have too significant an effect upon battery life. This is a trade-off that has triggered research in this area from an early stage, e.g. [1], [2], [3], [4], [5], [6], and more recent control-theoretic approaches as in [7], [8], [9]. A significant requirement in a large-scale network is the need for the control

This work was supported in part by the United Kingdom Engineering and Physical Sciences Research Council (EPSRC) and by the Royal Academy of Engineering.

Eoin Devane is with the Cambridge Centre for Analysis, Centre for Mathematical Sciences, University of Cambridge, Wilberforce Road, Cambridge, CB3 0WA, United Kingdom (e-mail: esmd2@cam.ac.uk).

Ioannis Lestas is with the Department of Engineering, University of Cambridge, Trumpington Street, Cambridge, CB2 1PZ, United Kingdom (email: icl20@cam.ac.uk). of the nodes' power allocations to be distributed, relying on local measurements of interference and battery life, as opposed to requiring a central control system. An important algorithm that controls power distributions for wireless networks in such a decentralized manner is the Foschini-Miljanic algorithm [2], which allows each user to update its transmitter power based only on local interference and signal power measurements at its corresponding receiver. The work [4] studied an important generalization of this, replacing the interference term with a nonlinearity satisfying certain generic properties. It was shown that this generalization allows a number of important single-channel uplink power control schemes, such as minimum power assignment [10], [11] and macrodiversity [12], to be analyzed in a unified way, while also admitting the incorporation of significant extensions such as the inclusion of saturation in the transmitted power. A number of variations of this framework have also been investigated, for instance in [13], [14]. A generalized class of continuous-time algorithms motivated by this work was studied in [15], where it was proved that, even if delays are present in the system, any fixed point is necessarily uniformly asymptotically stable.

Over recent years, there has been a proliferation of mobile use of wireless networks. When network users are in relative motion, the link gains between their respective transmitters and receivers may vary with time, giving an explicit timedependence to the corresponding mathematical model. This makes the study of time-dependent wireless networks both relevant and important.

In this paper, we consider a generalization of the system studied in [15] by allowing the system nonlinearity to depend explicitly on time. We consider first the undelayed case, and prove that any bounded power distribution solution is uniformly asymptotically stable and moreover that if such a bounded solution exists, then every solution has the same asymptotic behavior as $t \rightarrow \infty$. We then introduce arbitrary bounded delays into the model, and we show that any solution along which the nonlinearity is bounded is still uniformly asymptotically stable and further that if such a solution exists, then every solution will tend to it asymptotically as $t \rightarrow \infty$.

The paper is organized as follows. In section II we present a review of the theoretical results that will be needed in later sections. This includes a detailed overview of the theory of Lyapunov-Razumikhin functions for the study of stability properties of retarded functional differential equations. Section III presents the formulation of the problem to be considered. In section IV we state and prove our main results, for both the 
undelayed system and the delayed system. These are used in section $\mathrm{V}$ to derive time-invariant bounds on the asymptotic behavior of the trajectories when the generalized nonlinearities are appropriately constrained. Numerical simulations for a prototype model of a wireless network with users in relative motion are then presented in section VI and are shown to be in agreement with the theory in the previous sections. Finally, our conclusions are presented in section VII. The appendix considers an extension of the problem in which the static feedback gains are replaced by a more general class of passive feedback nonlinearities.

\section{BACKGROUND THEORY}

In this section, we review the key definitions and results that will be used in the rest of the paper.

Our first results will concern stability properties of undelayed systems of ordinary differential equations, for which we shall make use of the standard Lyapunov stability theory as presented in [16], [17]. We will then introduce delays into our system, casting the problem into the form of a retarded functional differential equation. We now review below some key elements of the theory of such equations, as detailed in [18].

Suppose that all delays (possibly heterogeneous and timevarying) present in the system are confined to lie in the interval $[0, r]$. Then, we let $\mathcal{C}\left([a, b], \mathbb{R}^{N}\right)$ be the Banach space of continuous functions mapping $[a, b]$ into $\mathbb{R}^{N}$, with elemental norm given by $\|\phi\|=\sup _{a \leq \theta \leq b}|\phi(\theta)|$, and we define $\mathcal{C}=\mathcal{C}\left([-r, 0], \mathbb{R}^{N}\right)$. If $\psi \in \mathcal{C}\left(\left[t_{0}-r, t_{0}+A\right], \mathbb{R}^{N}\right)$ for a given initial time $t_{0}$ and some $A>0$, and $t \in\left[t_{0}, t_{0}+A\right]$, then we define the delayed version of $\psi, \psi_{t} \in \mathcal{C}$, to be the segment of $\psi$ satisfying $\psi_{t}(\theta)=\psi(t+\theta)$ for $-r \leq \theta \leq 0$. The general form of the retarded functional differential equation to be considered is then

$$
\dot{x}(t)=f\left(t, x_{t}\right),
$$

where $x: \mathbb{R} \rightarrow \mathbb{R}^{N}, f: \mathbb{R} \times \mathcal{C} \rightarrow \mathbb{R}^{N}$, and the derivative being considered is the right-hand derivative. $x$ is a solution of (1) on $\left[t_{0}-r, t_{0}+A\right]$ for some $A>0$, with the initial condition $\phi \in \mathcal{C}$, if $x \in \mathcal{C}\left(\left[t_{0}-r, t_{0}+A\right], \mathbb{R}^{N}\right), x(t)$ satisfies (1) for $t \in$ $\left[t_{0}, t_{0}+A\right]$, and $x_{t_{0}}=\phi$ on $[-r, 0]$. In this case, we say that $x$ is a solution ${ }^{1}$ of (1) through $\left(t_{0}, \phi\right)$. When we want to make the initial data explicit, we will denote this solution by $x\left[t_{0}, \phi\right](t)$.

Analogously to the well-known stability definitions for undelayed systems [16], we say that the solution $x=X(t)$ is uniformly asymptotically stable if the following ${ }^{2}$ hold for all $t_{0} \geq 0$ :

i) given any $\epsilon>0$, there exists $\xi>0$, independent of $t_{0}$, such that $\left\|x_{t_{0}}-X_{t_{0}}\right\|<\xi$ implies $|x(t)-X(t)|<\epsilon$ for all $t>t_{0}$

ii) there exists $c>0$, independent of $t_{0}$, such that $\| x_{t_{0}}-$ $X_{t_{0}} \|<c$ implies $x(t)-X(t) \rightarrow 0$ uniformly as $t \rightarrow \infty$,

\footnotetext{
${ }^{1}$ A discussion on existence and uniqueness of solutions can be found in [18] Some basic sufficient conditions include continuity of $f$ for existence, and Lipschitz continuity of $f$ in $x_{t}$ for uniqueness, but much weaker conditions have also been derived in the literature.

${ }^{2}$ The notation $|\cdot|$ can represent any norm on $\mathbb{R}^{N}$.
}

for any other solution $x(t)$ of (1). If $\lim _{\epsilon \rightarrow \infty} \xi(\epsilon)=\infty$ and (ii) holds for any $c>0$, then we say that $x=X(t)$ is globally uniformly asymptotically stable.

Razumikhin [19] introduced the idea of considering a functional

$$
\bar{V}\left(t, x_{t}\right)=\sup _{-r \leq \theta \leq 0} V(t+\theta, x(t+\theta))
$$

and determining appropriate conditions on the $V$ here that allow this to play the same role for (1) as Lyapunov functions play for systems of ordinary differential equations. This theory employs the time-derivative along the system trajectory $x[t, \phi]$, defined as

$$
\begin{array}{r}
\dot{V}(t, \phi(0))=\limsup _{h \rightarrow 0+} \frac{1}{h}\{V(t+h, x[t, \phi](t+h)) \\
-V(t, \phi(0))\} .
\end{array}
$$

As is standard for stability analysis in the undelayed setting, we will transform our system to one with the zero solution as the image of the particular trajectory whose stability properties we wish to investigate. The following theorem ${ }^{3}$ gives conditions for $x=0$ to be uniformly asymptotically stable.

Theorem 1 (Razumikhin Theorem): Let $x=0$ be a solution of (1). Suppose that $f: \mathbb{R} \times \mathcal{C} \rightarrow \mathbb{R}^{N}$ in (1) takes $\mathbb{R} \times$ (bounded sets in $\mathcal{C}$ ) into bounded sets in $\mathbb{R}^{N}$, and $q, u$, $v$, $w: \overline{\mathbb{R}}_{+} \rightarrow \overline{\mathbb{R}}_{+}$are continuous, non-decreasing functions with $q(s)>s$ and $u(s), v(s), w(s)>0$ for all $s>0, u(0)=$ $v(0)=0$, and $v$ strictly increasing. Suppose further that there exists a continuous function $V: \overline{\mathbb{R}}_{+} \times \mathbb{R}^{N} \rightarrow \mathbb{R}$ such that:

i) $u(|x|) \leq V(t, x) \leq v(|x|), \forall t \in \overline{\mathbb{R}}_{+}, \forall x \in \mathbb{R}^{N}$,

ii) $\dot{V}(t, x(t)) \leq-w(|x(t)|)$ if $V(t+\theta, x(t+\theta)) \leq$ $q(V(t, x(t)))$ for all $\theta \in[-r, 0]$, where $x(t)$ is any trajectory of (1).

Then the solution $x=0$ is uniformly asymptotically stable.

If moreover $u(s) \rightarrow \infty$ as $s \rightarrow \infty$, then $x=0$ is globally uniformly asymptotically stable.

\section{PROBLEM FORMULATION}

We shall focus on the problem of controlling the power of the network users' uplink signals, that is the signals sent from the users' transmitting antennae to their corresponding receivers. Most commonly, the transmitters might be on-board the using devices, while the corresponding receivers would be in the form of local base stations. This model is illustrated in Fig. 1.

Let the transmitted power of user $i$ at time $t$ be given by $p_{i}(t)$, and define $p=\left(p_{1}, p_{2}, \ldots, p_{N}\right)^{T}$. Let the link gain between the transmitter of user $j$ and the receiver of user $i$ be $G_{i j}$ and the noise in the power received at user $i$ be $\nu_{i}$. At the receiver of user $i$, the received signal power from the transmitter of any other user $j \neq i$ is then equal to $G_{i j} p_{j}$. Consequently, summing over all received powers, the effective interference at the receiver of user $i$ is then given by

$$
R_{i}(p)=\frac{1}{G_{i i}}\left(\sum_{j \neq i} G_{i j} p_{j}+\nu_{i}\right),
$$

${ }^{3} \overline{\mathbb{R}}_{+}$denotes the closure of $\mathbb{R}_{+}=\{s \in \mathbb{R}: s>0\}$. 


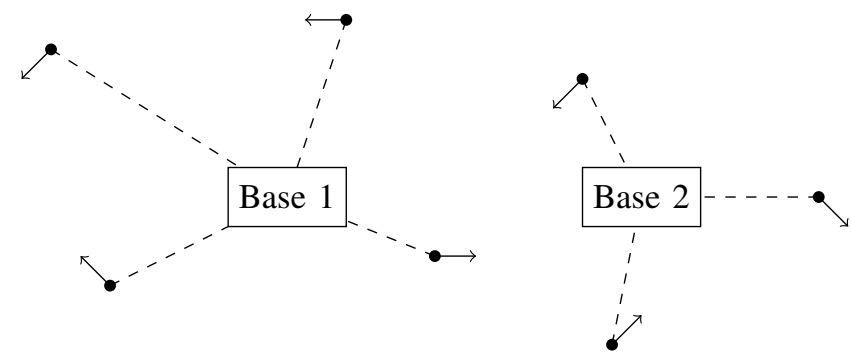

Fig. 1. Illustration of an example of the system under consideration. Users are in motion relative to a collection of base stations. We consider control of the power of the uplink signal from each user's transmitter, which is onboard the using device, to its corresponding receiver, which is within a base station. In situations as pictured in which there are multiple base stations, the base station acting as the receiver for each user is determined by some base station assignment rule.

and the signal-to-interference-ratio (SIR) is $\Gamma_{i}(p)=\frac{p_{i}}{R_{i}(p)}$. The continuous form of the Foschini-Miljanic algorithm [2] is

$$
\frac{d p_{i}(t)}{d t}=k_{i}\left(-p_{i}(t)+\gamma_{i} R_{i}(p)\right)
$$

where each $k_{i}$ is a positive constant. This scheme requires local measurements of the total received interference signal $\sum_{j \neq i} G_{i j} p_{j}+\nu_{i}$ and the received signal power $G_{i i} p_{i}$ to be made in the receiver of user $i$. These measurements are then relayed ${ }^{4}$ back to the transmitter of user $i$, enabling it to calculate a value for the effective interference $R_{i}(p)$. User $i$ is then able to update its transmission power completely autonomously according to its local equation (2). Consequently, this represents a distributed power update scheme. Moreover, it was shown in [2] that this algorithm is guaranteed to converge to a steady state power vector $p^{*}$ satisfying $p_{i}^{*}=\gamma_{i} R_{i}\left(p^{*}\right)$ at each node for a given SIR target $\gamma_{i}$, provided that such a power distribution exists. Motivated by the framework in [4], the stability properties of a generalized version of this system were studied in [15], where the $\gamma_{i} R_{i}(p)$ were replaced with a general class of nonlinear functions $I_{i}(p)$.

In wireless networks, however, the system gains are timevarying as the underlying network is changing in real-time. It is therefore important for this time-dependence of the system parameters to be reflected in corresponding mathematical formulations. In order to allow for these features we therefore extend the above framework by allowing the generalized nonlinearities to be time-dependent. In particular, we consider the system

$$
\frac{d p_{i}(t)}{d t}=k_{i}\left(-p_{i}(t)+I_{i}(t, p)\right),
$$

where $I(t, p)=\left(I_{1}(t, p), I_{2}(t, p) \ldots, I_{N}(t, p)\right)^{T}$ is required to satisfy the following properties at all times $t$ for all $p \geq 0:^{5}$

i) Monotonicity: if $p \geq p^{\prime}$, then $I(t, p) \geq I\left(t, p^{\prime}\right)$,

\footnotetext{
${ }^{4}$ The link gain $G_{i i}$ can be calculated by user $i$ by dividing the received power $G_{i i} p_{i}$ by the corresponding transmitted power $p_{i}$. It should be noted that in the presence of communication delays, the time at which the measurement of the received power was made will also need to be communicated if there is a significant change in the $G_{i i}$ during the delay period.

${ }^{5} \mathrm{By}$ vector inequalities, such as $p \geq p^{\prime}$ with $p, p^{\prime} \in \mathbb{R}^{N}$, we mean component-wise inequality.
}

ii) Scalability: there exists a continuous function $\delta$ : $(1, \infty) \rightarrow \mathbb{R}^{+}$such that, for any $\alpha>1, I_{i}(t, p)-$ $\frac{1}{\alpha} I_{i}(t, \alpha p) \geq \delta(\alpha)$ for all $i \in\{1,2, \ldots, N\}$.

In the absence of explicit time-variation, it was shown in [4] that, for many important single-channel interference-based uplink power control schemes, the effective interference can be represented by a nonlinearity satisfying such properties ${ }^{6}$. For instance, it was shown that power control schemes such as minimum-power assignment [10], [11] and macrodiversity [12] fit within this interference function framework, and this framework also allows the incorporation of important extensions such as the inclusion of saturation in the transmitted power. The specific measurements that are required here will clearly depend on the exact interference function under consideration. However, analogously to the discussion above, only measurements of the received interference and signal powers at the corresponding receiver will be required for user $i$ to update its transmission power. In particular, the individual transmission power of other users are not needed, meaning that power control algorithms of this form again represent distributed update schemes. It should be noted, however, that there are also limitations to the model. For instance, as discussed in [4], this framework is inapplicable for power control in multi-channel wireless networks and in the multiple base station downlink problem, as a result of the fact that the effective interference fails to satisfy the monotonicity property in this context.

By considering $t$ to be fixed, the proofs obtained in [4], [15] that monotonicity and scalability imply positivity and continuity results for $I$ in the absence of time-dependence can be seen to carry over into the present setting. Therefore, we have the following results for all $t$ :

Lemma 1: $I(t, p)>0$ for all $p \geq 0$.

Lemma 2: $I(t, p)$ is continuous in $p$ for all $p \geq 0$.

Invoking the uniformity of our scalability assumption, we now extend Lemma 1 to the following stronger result, valid at all times $t$.

Lemma 3: There exists a constant $c>0$ such that $I(t, p)>c$ for all $p \geq 0$.

Proof: According to the scalability and monotonicity assumptions, we have

$$
\begin{aligned}
\alpha I_{i}(t, p) & \geq I_{i}(t, \alpha p)+\alpha \delta(\alpha) \\
& \geq I_{i}(t, p)+\alpha \delta(\alpha)
\end{aligned}
$$

for all $\alpha>1, p \geq 0$, and $i \in\{1,2, \ldots, N\}$. Rearrangement of this gives a lower bound, valid for all $i \in\{1,2, \ldots, N\}$,

$$
I_{i}(t, p) \geq \sup _{\alpha>1} \frac{\alpha}{\alpha-1} \delta(\alpha)
$$

whence $c=\frac{1}{2} \sup _{\alpha>1} \frac{\alpha}{\alpha-1} \delta(\alpha)>0$ proves the result.

Remark 1: In principle, the distributed algorithm (3) requires a perfect measurement of the value of the generalized interference nonlinearity $I_{i}(t, p)$ to be made at the receiver and then communicated back to the transmitter for the update scheme

\footnotetext{
${ }^{6}$ The assumptions stated here may be thought of as uniform-in-time extensions of those presented in [4], where the model is introduced and its physical interpretation is discussed.
} 
to be applied. In general, the undertaking and communication of such a perfect measurement may not be possible due to factors such as measurement noise and packet loss ${ }^{7}$. These will inevitably lead to fluctuations in the transmitted powers. It should be noted, however, that such uncertainties in the value of $I_{i}(t, p)$ can also be regarded as introducing another source of time-variation into this quantity. Consequently, in cases where this uncertainty can be modeled as a bounded stochastic process, the analysis that follows will still be applicable to guarantee a stable system and provide bounds on the trajectories ${ }^{8}$. In situations where the measurement noise is more significant, the application of specific protocols, such as interference averaging ${ }^{9}$ and logarithmic interference averaging [4], may also be of value in reducing the fluctuations in the transmitted powers.

\section{RESULTS}

We begin by studying (3) in the absence of delays, before introducing into the framework delays which may be heterogeneous and time-dependent. In particular, we show in the undelayed case that if a bounded solution $p=P(t)$ exists then this is uniformly asymptotically stable. For the delayed case we show that if a solution $p=P(t)$ exists for which the generalized nonlinearity $I$ is bounded, then this is also uniformly asymptotically stable. In both cases the stability is also shown to be global, i.e. for all initial conditions all solutions $p(t)$ have the same asymptotic behavior.

\section{A. Undelayed system}

Theorem 2: Suppose that $p=P(t)$ is a bounded solution of (3) subject to the initial condition $P(0) \geq 0$. Then any trajectory $p(t)$ with $p(0) \geq 0$ will tend uniformly to $P(t)$ as $t \rightarrow \infty$.

Proof: First, notice that if $p(t)$ is any solution of (3) with some $p_{i}$ initially zero, then the positivity of $I_{i}(0,0)$ means that $p_{i}$ must be initially increasing, whence $p_{i}(t)>0$ for all $t>0$. Therefore, admitting a possible shift in the time-origin in order to deal with such trajectories, it will suffice in what follows to explicitly consider only trajectories with strictly positive initial conditions. Thus, we may henceforth suppose that we have $p(0)>0$ for all trajectories and in particular also $P(0)>0$.

We note now that, according to Lemma 3 , if $p_{i}(T) \leq c$ ever occurs for an arbitrary trajectory, then (3) gives

$$
\frac{d p_{i}(T)}{d t}>0
$$

\footnotetext{
${ }^{7}$ Packet dropouts are not explicitly addressed within the paper, however, it should be noted that in specific protocols, such as ones where the latest available information is used to implement the control policy, the feedback scheme could be modeled by means of time-varying delays and would hence fit within the framework analyzed in the paper.

${ }^{8}$ Note that when an additive time-varying noise term is added to $I(t, p)$, i.e. $I^{\prime}(t, p)=I(t, p)+w(t)$, then $I^{\prime}$ retains the monotonicity and scalability properties of $I$. See also sections $V$ and VI where time invariant bounds on the trajectories are derived when $I$ is appropriately bounded.

${ }^{9}$ Interference averaging can be shown to be equivalent in continuous time to a reduction of the gain $k_{i}$. Note, however, that there is a tradeoff between disturbance rejection and speed of response when $k_{i}$ is reduced (this is also discussed in section VI).
}

Therefore, since the initial data are assumed to be strictly positive and the system trajectories are continuous, we obtain the strictly positive lower bound $p(t) \geq \min \left\{c, \min _{i} p_{i}(0)\right\}>0$ for all $t \geq 0$. This allows us to make the change of variables

$$
\pi_{i}=\log \left(\frac{p_{i}}{P_{i}}\right)
$$

in (3). This reduces the equation to ${ }^{10}$

$$
\frac{d \pi_{i}}{d t}=\frac{k_{i}}{P_{i} e^{\pi_{i}}}\left[I_{i}\left(t, \operatorname{diag}\left(e^{\pi_{j}}\right) P\right)-e^{\pi_{i}} I_{i}(t, P)\right]
$$

and transforms the solution $p=P(t)$ to an equilibrium of the new system (5) at $\pi=0$, allowing us to invoke classical Lyapunov stability theory. We will consider the candidate Lyapunov function

$$
V(\pi)=\max _{i}\left|\pi_{i}\right|,
$$

which is a continuous, positive-definite function of $\pi$ alone with derivative given by

$$
\frac{d}{d t} V(\pi)= \begin{cases}\dot{\pi}_{i_{m}(t)}(t) & \text { if } \pi_{i_{m}(t)}(t)>0, \\ -\dot{\pi}_{i_{m}(t)}(t) & \text { if } \pi_{i_{m}(t)}(t)<0, \\ 0 & \text { if } \pi_{i_{m}(t)}(t)=0,\end{cases}
$$

where $i_{m}$ is defined to satisfy ${ }^{11}\left|\pi_{i_{m}}\right| \geq\left|\pi_{j}\right|$ for all $j$. The final case here occurs if and only if $\pi=0$. There remains a dichotomy to consider. We simplify the notation here by writing $i$ for $i_{m}$.

a) If $\pi_{i}>0$, then $e^{\pi_{i}}>1$ and $\pi_{i} \geq \pi_{j}$ for all $j$, so that the properties of monotonicity and scalability give

$$
\begin{aligned}
e^{\pi_{i}} I_{i}(t, P) & \geq I_{i}\left(t, e^{\pi_{i}} P\right)+e^{\pi_{i}} \delta\left(e^{\pi_{i}}\right) \\
& \geq I_{i}\left(t, \operatorname{diag}\left(e^{\pi_{j}}\right) P\right)+e^{\pi_{i}} \delta\left(e^{\pi_{i}}\right) .
\end{aligned}
$$

b) If $\pi_{i}<0$, then $e^{\pi_{i}}<1$ and $\pi_{i} \leq \pi_{j}$ for all $j$, so that the properties of monotonicity and scalability give

$$
\begin{aligned}
I_{i}\left(t, \operatorname{diag}\left(e^{\pi_{j}}\right) P\right) & \geq e^{\pi_{i}} I_{i}\left(t, e^{-\pi_{i}} \operatorname{diag}\left(e^{\pi_{j}}\right) P\right)+\delta\left(e^{-\pi_{i}}\right) \\
& \geq e^{\pi_{i}} I_{i}\left(t, e^{-\pi_{i}} e^{\pi_{i}} P\right)+e^{\pi_{i}} \delta\left(e^{-\pi_{i}}\right) \\
& =e^{\pi_{i}} I_{i}(t, P)+e^{\pi_{i}} \delta\left(e^{-\pi_{i}}\right) .
\end{aligned}
$$

Therefore, if we put $\kappa=\min _{j} k_{j}$ and let $B$ be an upper bound for $P,(5)$ and (6) give

$$
\frac{d}{d t} V(\pi) \begin{cases}\leq-\frac{\kappa \delta\left(e^{V(\pi)}\right)}{B} & \text { if some } \pi_{j} \neq 0, \\ =0 & \text { if } \pi=0 .\end{cases}
$$

The right-hand side here is then a continuous, negative-definite function of $\pi$ alone. Thus, all the conditions of the Lyapunov theorem are satisfied, meaning that $\pi=0$ is a uniformly asymptotically stable equilibrium of (5).

Furthermore, the Lyapunov function $V(\pi)=\max _{i}\left|\pi_{i}\right|$ is radially unbounded, meaning that the above in fact shows $\pi=0$ to be a globally uniformly asymptotically stable equilibrium of (5) and hence that every trajectory $p(t)$ of

\footnotetext{
${ }^{10} \operatorname{diag}\left(e^{\pi_{j}}\right)$ means the $N \times N$ matrix with entries $e^{\pi_{1}}, e^{\pi_{2}}, \ldots, e^{\pi_{N}}$ on the leading diagonal and zeros everywhere else.

${ }^{11}$ In the event that this is satisfied by more than one value of $i_{m}$, we take $i_{m}$ as the one with maximal $\frac{d\left|\pi_{i}\right|}{d t}$. If this still does not give a unique value, then any of the remaining possibilities can be used.
} 
(3) with $p(0)>0$ will tend uniformly to $P(t)$ as $t \rightarrow \infty$. Recalling our comment from the beginning of the proof about how to deal with trajectories with initial conditions possibly zero, we see that this completes the proof.

\section{B. Delayed system}

We now consider a further generalization of (3) by incorporating delays into the system. Thus, we allow the interference function $I$ to depend on not just the current power states, but also those at earlier times. Specifically, we consider the delayed system

$$
\frac{d p_{i}(t)}{d t}=k_{i}\left(-p_{i}(t)+I_{i}\left(t, p^{d_{i}}(t)\right)\right),
$$

where

$p^{d_{i}}(t)=\left(p_{1}\left(t-\theta_{i 1}(t)\right), p_{2}\left(t-\theta_{i 2}(t)\right), \ldots, p_{N}\left(t-\theta_{i N}(t)\right)\right)^{T}$, with all delays $\theta_{i j}$ restricted to lie in some interval $[0, r]$.

We also assume the previously stated properties of $I$ and additionally require in the scalability condition that $\delta$ be nondecreasing.

The system is then amenable to stability analysis using the methods of Razumikhin detailed in section II.

Theorem 3: Suppose that $p=P(t)$ is a solution of equation (7) satisfying $P(\theta) \geq 0$ for all $\theta \in[-r, 0]$. Suppose further that there exists a positive constant $C$ such that $I_{i}\left(t, P^{d_{i}}(t)\right)<C$ for all $i \in\{1,2, \ldots, N\}$ and all $t \geq 0$. Then any trajectory $p(t)$ with $p(\theta) \geq 0$ for $\theta \in[-r, 0]$ will tend uniformly to $P(t)$ as $t \rightarrow \infty$.

Proof: Analogously to the approach in the proof of Theorem 2, we observe that if $p(t)$ is any solution of (7) with initial condition satisfying the weak inequality $p(\theta) \geq 0$ for all $\theta \in[-r, 0]$, then the lower-boundedness property of Lemma 3 implies that, for all $i \in\{1,2, \ldots, N\}, \frac{d p_{i}(t)}{d t}>0$ whenever $p_{i}(t) \leq c$. This means that we must have $p(t)>0$ for all $t>0$, and so if we shift the time-origin to any point in excess of the maximal delay $r$, then all initial conditions of $p$ will be strictly positive. Therefore, admitting this shift in time-origin in order to deal with such trajectories, we may henceforth assume that all trajectories (in particular including $P(t)$ ) satisfy $p(\theta)>0$ for all $\theta \in[-r, 0]$.

As in the proof of Theorem $2, \min \left\{c, \min _{i} p_{i}(0)\right\}$ consequently becomes a strictly positive lower bound for every component of any trajectory $p(t)$ for all $t \geq 0$.

In addition, we have the hypothesis that $I_{i}\left(t, P^{d_{i}}(t)\right)<C$ for all $i \in\{1,2, \ldots, N\}$ and all $t \geq 0$. Then, if $P_{i}(t) \geq C$, (7) gives $\frac{d P_{i}(t)}{d t}<0$, whence $B:=2 \max \left\{C, \max _{i} P_{i}(0)\right\}>0$ is a strict upper bound for every component of $P(t)$ for all $t \geq 0$.

The lower bound on each $p_{i}(t)$ allows us again to make the substitution

$$
\pi_{i}=\log \left(\frac{p_{i}}{P_{i}}\right)
$$

yielding the new equation

$$
\frac{d \pi_{i}}{d t}=\frac{k_{i}}{P_{i} e^{\pi_{i}}}\left[I_{i}\left(t, \operatorname{diag}\left(e^{\pi_{j}^{d_{i}}}\right) P^{d_{i}}\right)-e^{\pi_{i}} I_{i}\left(t, P^{d_{i}}\right)\right]
$$

and transforming the particular solution $p=P(t)$ to the solution $\pi=0$. The system is then in a form to which we may apply Theorem 1 .

Define a candidate Lyapunov-Razumikhin function for the $t \geq 0$ system as

$$
V(\pi)=\max _{i}\left|\pi_{i}\right| .
$$

This is continuous and satisfies condition (i) of Theorem 1 by virtue of the fact that $V(t, x)=\|x\|_{\infty}$.

In order to satisfy condition (ii) of Theorem 1, we shall consider $q(s)=s+f(s)$, where $f$ is assumed nonnegative and is to be determined. Then we suppose that

$$
q(V(t, \pi(t))) \geq \sup _{-r \leq \theta \leq 0} V(t+\theta, \pi(t+\theta)),
$$

whence, for all $j \in\{1,2, \ldots, N\}$,

$$
\begin{aligned}
q\left(\left|\pi_{i_{m}(t)}(t)\right|\right) & \geq \sup _{-r \leq \theta \leq 0}\left|\pi_{i_{m}(t+\theta)}(t+\theta)\right| \\
& \geq\left|\pi_{j}^{d_{i_{m}(t)}}\right| .
\end{aligned}
$$

Similarly to before, we have ${ }^{12}$

$$
\frac{d V}{d t}= \begin{cases}\dot{\pi}_{i_{m}(t)}(t) & \text { if } \pi_{i_{m}(t)}(t)>0, \\ -\dot{\pi}_{i_{m}(t)}(t) & \text { if } \pi_{i_{m}(t)}(t)<0, \\ 0 & \text { if } \pi_{i_{m}(t)}(t+\theta)=0, \forall \theta \in[-r, 0],\end{cases}
$$

and the third case is trivial, leaving a dichotomy to consider. Again, write $i$ for $i_{m}$ here to reduce the notational complexity.

i) Suppose that $\pi_{i}>0$. Inequality (10) gives $\pi_{j}^{d_{i}}-q\left(\pi_{i}\right) \leq$ 0 , whence monotonicity gives

$$
\begin{aligned}
I_{i}(t, \operatorname{diag} & \left.\left(e^{\pi_{j}^{d_{i}}}\right) P^{d_{i}}\right) \\
& =I_{i}\left(t, e^{q\left(\pi_{i}\right)} \operatorname{diag}\left(e^{\pi_{j}^{d_{i}}-q\left(\pi_{i}\right)}\right) P^{d_{i}}\right) \\
& \leq I_{i}\left(t, e^{q\left(\pi_{i}\right)} P^{d_{i}}\right) \\
& \leq e^{q\left(\pi_{i}\right)}\left(I_{i}\left(t, P^{d_{i}}\right)-\delta\left(e^{q\left(\pi_{i}\right)}\right)\right) \\
& \leq e^{q\left(\pi_{i}\right)}\left(I_{i}\left(t, P^{d_{i}}\right)-\delta\left(e^{\pi_{i}}\right)\right)
\end{aligned}
$$

by scalability, using the fact that $\delta$ is now assumed to be non-decreasing. Suppose now that $f \leq f_{1}$ for some continuous function $f_{1}$ satisfying $f_{1}(0)=0$ and $f_{1}(s)>$ 0 for $s>0$. Then (8) gives

$$
\dot{\pi}_{i} \leq \frac{k_{i}}{P_{i}}\left[I_{i}\left(t, P^{d_{i}}\right)\left(e^{f_{1}\left(\pi_{i}\right)}-1\right)-e^{f_{1}\left(\pi_{i}\right)} \delta\left(e^{\pi_{i}}\right)\right] .
$$

Now recall the bound on $I_{i}\left(t, P^{d_{i}}(t)\right)$. This gives

$$
\dot{\pi}_{i}<\frac{k_{i}}{P_{i}}\left[C\left(e^{f_{1}\left(\pi_{i}\right)}-1\right)-\delta\left(e^{\pi_{i}}\right)\right],
$$

since $e^{f_{1}\left(\pi_{i}\right)}>1$. Suppose that

$$
1<e^{f_{1}\left(\pi_{i}\right)}<1+\frac{\delta\left(e^{\pi_{i}}\right)}{2 C} .
$$

Defining $\kappa=\min _{j} k_{j}$ and recalling the upper bound on $P_{i}(t)$, (11) then gives

$$
\dot{\pi}_{i}<-\frac{\kappa \delta\left(e^{\pi_{i}}\right)}{2 B}
$$

${ }^{12}$ Note that the value of $\frac{d V}{d t}$ when $\pi_{i_{m}}(t)=0$ and $\pi_{i_{m}}(t+\theta) \neq 0$ for some $\theta \in[-r, 0)$ is not needed in order to deduce stability using Theorem 1 . 
But we note now that $\lim _{\pi_{i} \downarrow 0} \delta\left(e^{\pi_{i}}\right)=0$ and $\delta\left(e^{\pi_{i}}\right)$ is non-decreasing as a function of $\pi_{i}$. This makes the righthand sides of the preceding inequalities, respectively, non-decreasing and non-increasing. Consequently, there exists a continuous, non-decreasing function (we may choose any such function) $f_{1}$ satisfying the properties required above such that

$$
1<e^{f_{1}\left(\pi_{i}\right)}<1+\frac{\delta\left(e^{\pi_{i}}\right)}{2 C},
$$

and a continuous, non-increasing function (again, any of the possibilities may be chosen) $y_{1}$ such that

$$
-\frac{\kappa \delta\left(e^{\pi_{i}}\right)}{2 B} \leq y_{1}\left(\pi_{i}\right)<0 .
$$

Then $f_{1}$ satisfies the foregoing argument, and we therefore get the required result that $\dot{\pi}_{i}<y_{1}\left(\pi_{i}\right)<0$.

ii) The $\pi_{i}<0$ case is similar. Inequality (10) gives $\pi_{j}^{d_{i}}+$ $q\left(-\pi_{i}\right) \geq 0$. Therefore

$$
\begin{aligned}
I_{i}(t, \operatorname{diag} & \left.\left(e^{\pi_{j}^{d_{i}}}\right) P^{d_{i}}\right) \\
& =I_{i}\left(t, e^{-q\left(-\pi_{i}\right)} \operatorname{diag}\left(e^{\pi_{j}^{d_{i}}+q\left(-\pi_{i}\right)}\right) P^{d_{i}}\right) \\
& \geq I_{i}\left(t, e^{-q\left(-\pi_{i}\right)} P^{d_{i}}\right) \\
& \geq e^{-q\left(-\pi_{i}\right)} I_{i}\left(t, P^{d_{i}}\right)+\delta\left(e^{q\left(-\pi_{i}\right)}\right) \\
& \geq e^{-q\left(-\pi_{i}\right)} I_{i}\left(t, P^{d_{i}}\right)+\delta\left(e^{-\pi_{i}}\right) .
\end{aligned}
$$

Then, assuming that $f \leq f_{2}$, with $f_{2}$ satisfying the same properties as $f_{1}$, and invoking the bounds introduced in case (i), we get

$$
\begin{aligned}
\dot{\pi}_{i} & \geq \frac{k_{i}}{P_{i}}\left[I_{i}\left(t, P^{d_{i}}\right)\left(e^{-f_{2}\left(-\pi_{i}\right)}-1\right)+e^{-\pi_{i}} \delta\left(e^{-\pi_{i}}\right)\right] \\
& >\frac{k_{i}}{P_{i}}\left[C\left(e^{-f_{2}\left(-\pi_{i}\right)}-1\right)+\delta\left(e^{-\pi_{i}}\right)\right] .
\end{aligned}
$$

Analogously to the above, we may then choose $f_{2}$ continuous and non-decreasing with

$$
1-\frac{\delta\left(e^{-\pi_{i}}\right)}{2 C}<e^{-f_{2}\left(-\pi_{i}\right)}<1,
$$

whence

$$
\dot{\pi}_{i}>\frac{\kappa \delta\left(e^{-\pi_{i}}\right)}{2 B}
$$

and we can introduce a continuous, non-decreasing function $y_{2}$ (non-decreasing here as the argument inherits a minus sign) such that

$$
0<y_{2}\left(-\pi_{i}\right) \leq \frac{\kappa \delta\left(e^{-\pi_{i}}\right)}{2 B} .
$$

Then we get $\dot{\pi}_{i}>y_{2}\left(-\pi_{i}\right)>0$.

To complete this stage of the proof we now define, for $s \geq 0$, the functions $q(s)=s+f(s)$, where $f(s)=$ $\min \left\{f_{1}(s), f_{2}(s)\right\}$, and $w(s)=\min \left\{-y_{1}(s), y_{2}(s)\right\}$. Then $q(0)=w(0)=0, q(s)>s$ for $s>0, q$ and $w$ are both continuous and non-decreasing, and $\dot{V} \leq-w\left(\left|\pi_{i_{m}}\right|\right)$ whenever (9) holds. Invoking our earlier observation that $\left|\pi_{i_{m}}\right|=\|\pi\|_{\infty}$ and the equivalence properties for norms on $\mathbb{R}^{N}$, we see that condition (ii) of Theorem 1 is satisfied, and so we conclude that the solution $\pi=0$ is uniformly asymptotically stable.

Furthermore, as noted in the proof of Theorem 2, $V(\pi)=$ $\max _{i}\left|\pi_{i}\right|$ is radially unbounded, meaning that the proof above in fact shows $\pi=0$ to be a globally uniformly asymptotically stable equilibrium of (8). Therefore, every trajectory $p(t)$ with $p(\theta)>0$ for all $\theta \in[-r, 0]$ will tend uniformly to $P(t)$ as $t \rightarrow \infty$. Recalling our observation from the beginning of the proof concerning how to incorporate trajectories with initial conditions possibly equal to zero within this framework, we see that this completes the proof.

Remark 2: The above results are all seen to be independent of the value of the $k_{i}$. This fact is a consequence of the continuous-time formulation of the algorithm that is being considered; in a discrete-time formulation, too large a value of some $k_{i}$ could give rise to instabilities. However, it would be expected that, for sufficiently small choices of the $k_{i}$, the corresponding discrete-time algorithm will exhibit convergent behavior in both the undelayed and delayed regimes.

\section{AsYMPTOTIC BOUNDS}

One important consequence of the results in section IV is that if one can find a bounded invariant set for the trajectories, then one can deduce that, for all initial conditions, the trajectories will converge to this set. This is used below to derive time-invariant asymptotic bounds for the power $p$ when the generalized nonlinearities satisfy appropriate bounds.

In particular, we let $I(t, p)$ satisfy both monotonicity and scalability, and we suppose in addition that there exist timeindependent nonlinearities $J^{\text {min }}$ and $J^{\text {max }}$ satisfying monotonicity and scalability, with

$$
J^{\min }(p) \leq J^{\max }(p)
$$

for all $p \geq 0$, such that the systems defined by

$$
\frac{d p_{i}(t)}{d t}=k_{i}\left(-p_{i}(t)+J_{i}(p)\right)
$$

for $J=J^{\min }$ and $J=J^{\max }$ have equilibria $q^{\min } \geq 0$ and $q^{\max } \geq 0$ respectively (i.e. $q^{\min }=J^{\min }\left(q^{\min }\right)$ and $\left.q^{\max }=J^{\max }\left(q^{\max }\right)\right)$. These equilibria are then unique (e.g. by [15]; also follows from Theorem 3 ). Suppose further that

$$
J^{\min }\left(q^{\min }\right)<I\left(t, q^{\min }\right) \text { and } I\left(t, q^{\max }\right)<J^{\max }\left(q^{\max }\right)
$$

hold $^{13}$ for all $t \geq 0$. Under these conditions, we obtain the following result ${ }^{14}$.

Proposition 1: The set

$$
\mathcal{D}=\left\{p: q^{\min } \leq p \leq q^{\max }\right\}
$$

is positively invariant with respect to (7), in the sense that if $p(\theta) \in \mathcal{D}$ for all $\theta \in[-r, 0]$, then $p(t) \in \mathcal{D}$ for all $t \geq 0$.

Consequently, any trajectory $p(t)$ must satisfy $p(t) \rightarrow \mathcal{D}$ as $t \rightarrow \infty$.

\footnotetext{
${ }^{13}$ Note that condition (13) implies, by monotonicity, that the nonlinearity satisfies the bound $J^{\min }(p)<I(t, p)<J^{\max }(p)$ for all $p \in \mathcal{D}$, where $\mathcal{D}=$ $\left\{p: q^{\min } \leq p \leq q^{\max }\right\}$ is the invariant region used in Proposition 1 below.

${ }^{14}$ The proposition is stated for the delayed system with the understanding that the corresponding result for the undelayed case can be obtained by letting the maximal delay $r=0$.
} 
Proof: We first show that $\mathcal{D}$ is non-empty. According to monotonicity and (12), we have

$$
J^{\min }(q) \leq J^{\min }\left(q^{\max }\right) \leq J^{\max }\left(q^{\max }\right)=q^{\max }
$$

for all $q \in\left[0, q^{\max }\right]$. Therefore, $J^{\min } \operatorname{maps}\left[0, q^{\max }\right]$ into $\left[0, q^{\max }\right]$, whence we may deduce, according to Brouwer's fixed point theorem, that $J^{\min }$ has a fixed point in $\left[0, q^{\max }\right]$. By uniqueness, this must be $q^{\min }$. Therefore, we have $q^{\min } \leq q^{\max }$, and thus $\mathcal{D}$ is non-empty.

Now we show that $\mathcal{D}$ is a positively invariant set for our system. Suppose that $T \geq 0$ is such that $p(T) \in \partial \mathcal{D}$ and $p(t) \in \mathcal{D}$ for all $t \in[0, T)$. There are then, incorporating the given initial conditions, two cases:

i) $p_{i}(T)=q_{i}^{\max }$ for some component $i$, and $p(t) \leq q^{\max }$ for all $t \in[-r, T]$. Then monotonicity and (13) give

$$
\begin{aligned}
\frac{d p_{i}(T)}{d t} & =k_{i}\left(-p_{i}(T)+I_{i}\left(T, p^{d_{i}}(T)\right)\right) \\
& \leq k_{i}\left(-q_{i}^{\max }+I_{i}\left(T, q^{\max }\right)\right) \\
& <k_{i}\left(-q_{i}^{\max }+J_{i}^{\max }\left(q^{\max }\right)\right) \\
& \left.=k_{i}\left(-q_{i}^{\max }+q_{i}^{\max }\right)\right) \\
& =0
\end{aligned}
$$

Therefore, the right-derivative of $p_{i}$ is strictly negative at time $T$, meaning that the value of $p_{i}$ must be decreasing. Consequently, since all trajectories are everywhere continuous and right-differentiable, any solution subject to $p(\theta) \in \mathcal{D}$ for all $\theta \in[-r, 0]$ must satisfy $p(t) \leq q^{\max }$ for all $t \geq 0$.

ii) $p_{i}(T)=q_{i}^{\text {min }}$ for some component $i$, and $p(t) \geq q^{\text {min }}$ for all $t \in[-r, T]$. Similarly to the first case, then

$$
\begin{aligned}
\frac{d p_{i}(T)}{d t} & =k_{i}\left(-p_{i}(T)+I_{i}\left(T, p^{d_{i}}(T)\right)\right) \\
& \geq k_{i}\left(-q_{i}^{\min }+I_{i}\left(T, q^{\min }\right)\right) \\
& >k_{i}\left(-q_{i}^{\min }+J_{i}^{\min }\left(q^{\min }\right)\right) \\
& \left.=k_{i}\left(-q_{i}^{\min }+q_{i}^{\min }\right)\right) \\
& =0
\end{aligned}
$$

Thus, we now have $p_{i}$ increasing at $T$, from which we may infer by the continuity and right-differentiability properties that any solution with $p(\theta) \in \mathcal{D}$ for all $\theta \in[-r, 0]$ must satisfy $p(t) \geq q^{\min }$ for all $t \geq 0$.

In combination, these two cases show that any trajectory subject to the initial condition $p(\theta) \in \mathcal{D}$ for all $\theta \in[-r, 0]$ necessarily satisfies $p(t) \in \mathcal{D}$ for all $t \geq 0$. Consequently, we have shown that $\mathcal{D}$ is a non-empty, positively invariant set. Therefore, we may specify initial conditions in $\mathcal{D}$, of the form $P(\theta) \in \mathcal{D}$ for all $\theta \in[-r, 0]$, and the resulting trajectory $p=P(t)$ must always remain in $\mathcal{D}$. But then this trajectory satisfies $P^{d_{i}}(t) \leq q^{\max }$, whence monotonicity and (13) yield

$$
I_{i}\left(t, P^{d_{i}}(t)\right) \leq I\left(t, q^{\max }\right)<J^{\max }\left(q^{\max }\right)=q^{\max },
$$

which is a constant, for all $i \in\{1,2, \ldots, N\}$ and all $t \geq 0$. Therefore, each $I_{i}\left(t, P^{d_{i}}(t)\right)$ is bounded and so Theorem 3 is applicable, telling us that every trajectory of this system $p(t)$ must tend asymptotically to $P(t)$ as $t \rightarrow \infty$. But, since $P(t) \in \mathcal{D}$ for all $t \geq 0$, this means that every trajectory satisfies $p(t) \rightarrow \mathcal{D}$ as $t \rightarrow \infty$.
Remark 3: The fact that $D$ is shown to be a non-empty, positively invariant set for the class of systems considered implies the existence of trajectories along which the system nonlinearity is bounded, thereby allowing us to ascertain that the convergence results of section IV apply (see also the example in the next section). Furthermore, the fact that the construction of invariant sets allows one to deduce global convergence to this set is an example of the strength of the results in the previous section, in that they allow one to predict asymptotic properties of all trajectories by simply studying the behavior of a convenient class of trajectories.

\section{EXAMPLES}

As a numerical example of the results in section IV, we simulate a time-varying version of the delayed form of the Foschini-Miljanic algorithm,

$$
\frac{d p_{i}(t)}{d t}=k_{i}\left(-p_{i}(t)+\frac{\gamma_{i}}{G_{i i}}\left(\sum_{j \neq i} G_{i j} p_{j}^{d_{i}}(t)+\nu_{i}\right)\right) .
$$

Consider the situation in which the users of a wireless network are in motion with respect to each other. The consequence is that, in general, all the separation distances between the users' transmitters and receivers will be constantly changing. Therefore, we expect the link gains $G_{i j}$ to depend on time. For all pairs $i, j \in\{1,2, \ldots, N\}$, we incorporate here this variation in the gains as a random process given by

$$
G_{i j}(t)=G_{i j}(0)\left(1+c W_{i j}(t)\right),
$$

where $W_{i j}(t)$ are independent standard Wiener processes and $c$ is a constant that depends on the environment and the speed of movement of the users.

In order to simulate the system we replace $c W_{i j}(t)$ by the linear interpolant of a discretized version of this process with step size $\tau$. Additionally, saturation is included in the gain values, which ensures that these remain positive and bounded. That is, we model the gain evolution as

$$
G_{i j}(t)=G_{i j}(0)\left(1+c S_{i j}(t)\right),
$$

where $S_{i j}(t)$ is a linear interpolation of values $\tilde{W}_{i j}\left(t_{k}\right)$ given by

$\tilde{W}_{i j}\left(t_{k+1}\right)=\min \left(\max \left(\tilde{W}_{i j}\left(t_{k}\right)+\tau N_{i j}(k), \frac{-0.49}{c}\right), \frac{0.49}{c}\right)$.

with $\tau=t_{k+1}-t_{k}$ and $N_{i j}(k)$ denoting independent standard normal random variables. For the simulations that follow, we shall take $\tau=10^{-2}$.

The system nonlinearity in (14) is therefore given by

$$
\begin{aligned}
& I_{i}(t, p) \\
& =\frac{\gamma_{i}}{G_{i i}(0)\left(1+c S_{i i}(t)\right)}\left(\sum_{j \neq i} G_{i j}(0)\left(1+c S_{i j}(t)\right) p_{j}+\nu_{i}\right) .
\end{aligned}
$$

Since the coefficients of the components $p_{j}$ are all positive, this clearly satisfies the monotonicity conditions. Moreover,

$$
I_{i}(t, p)-\frac{1}{\alpha} I_{i}(t, \alpha p)=\frac{\gamma_{i} \nu_{i}}{G_{i i}(0)\left(1+c S_{i i}(t)\right)}\left(1-\frac{1}{\alpha}\right),
$$




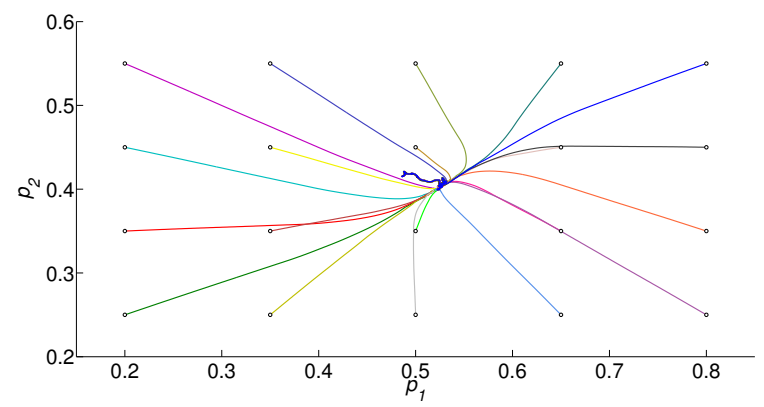

(a) $p_{2}$ against $p_{1}$ with the other initial conditions set to 0.4 .

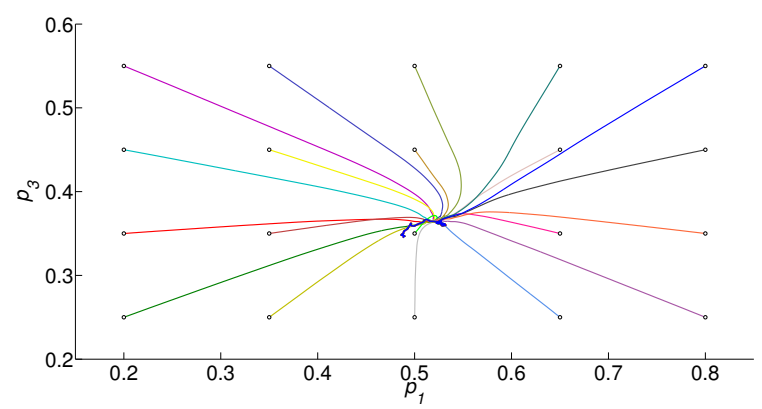

(c) $p_{3}$ against $p_{1}$ with the other initial conditions set to 0.4 .

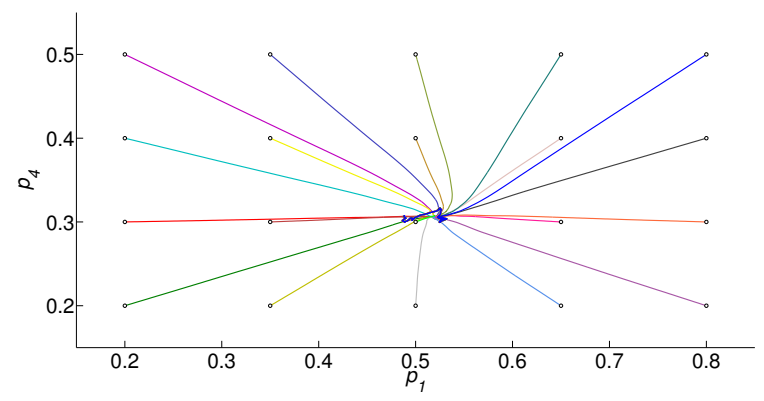

(e) $p_{4}$ against $p_{1}$ with the other initial conditions set to 0.4 .

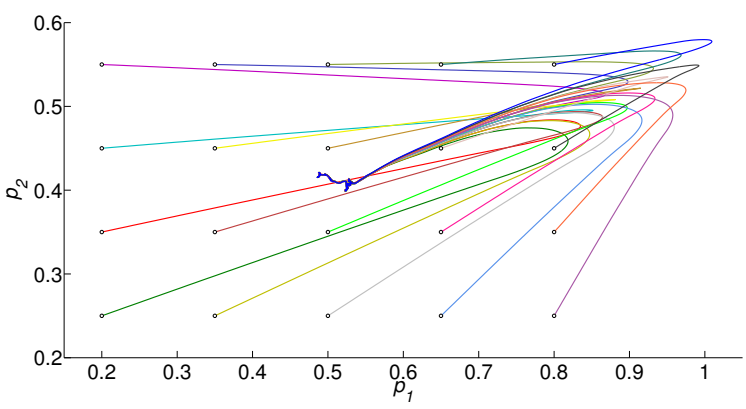

(b) $p_{2}$ against $p_{1}$ with the other initial conditions set to 2 .

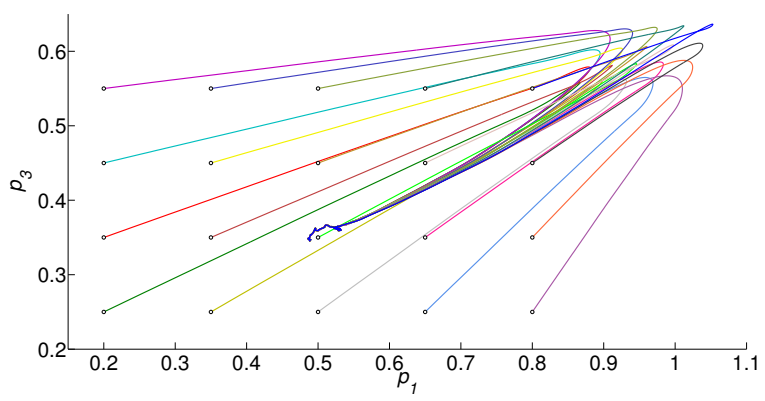

(d) $p_{3}$ against $p_{1}$ with the other initial conditions set to 2 .

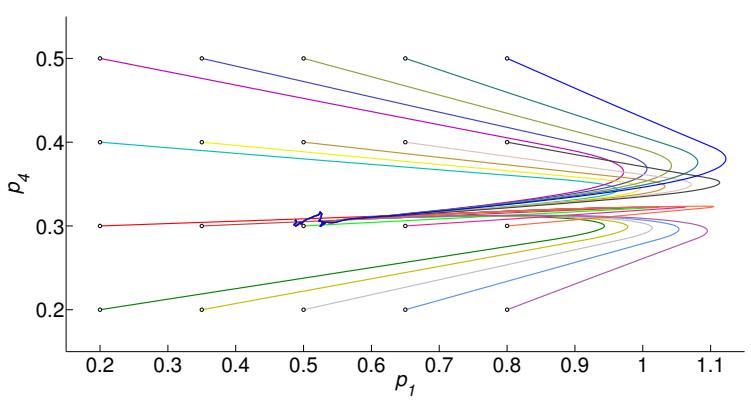

(f) $p_{4}$ against $p_{1}$ with the other initial conditions set to 2 .

Fig. 2. Two-dimensional projections of various solutions of system (14) with gain evolution (15) for different initial conditions.

which is strictly positive, continuous and non-decreasing as a function of $\alpha>1$. Consequently, defining $\delta(\alpha)$ to be equal to the right-hand side here, this nonlinearity is seen to also satisfy the scalability assumption with non-decreasing $\delta$.

With a view to applying Proposition 1, let us also define the time-independent nonlinearities

$$
\begin{aligned}
J_{i}^{\min }(p) & =\frac{\gamma_{i}}{G_{i i}(0)}\left(\sum_{j \neq i} \frac{1}{3} G_{i j}(0) p_{j}+\frac{2}{3} \nu_{i}\right), \\
J_{i}^{\max }(p) & =\frac{\gamma_{i}}{G_{i i}(0)}\left(\sum_{j \neq i} 3 G_{i j}(0) p_{j}+2 \nu_{i}\right) .
\end{aligned}
$$

By analogous argument to that above for $I_{i}(t, p)$, these clearly both satisfy the monotonicity and scalability conditions, while the saturation values in the definition of $S$ imply that

$$
J^{\min }(p) \leq I(t, p) \leq J^{\max }(p)
$$

for all $p \geq 0$ and all $t \geq 0$, with equality if and only if $p=0$.
Having introduced these quantities, we now specify the numerical values to be used for the simulations presented in Figs. 2, 3, 4, 5, and 6. We consider a simple example where the network comprises four users. For the parameters present, we take the values $c=0.0326, k_{i}=0.2, \gamma_{i}=6, \frac{\nu_{i}}{G_{i i}(0)}=0.05$,

$$
\frac{G_{i j}(0)}{G_{i i}(0)}=\left(\begin{array}{cccc}
1 & 0.0417 & 0.0341 & 0.0217 \\
0.0247 & 1 & 0.0042 & 0.0113 \\
0.0033 & 0.0012 & 1 & 0.0292 \\
0.0008 & 0.0003 & 0.0021 & 1
\end{array}\right) .
$$

These initial relative link gain values were computed for randomly chosen initial relative spatial locations of the users, assuming a path loss exponent of 2 throughout the ambient space. The value of the scaling constant $c$ has been chosen in order that the stochastic link gain variations be representative of those that might arise due to perambulatory motion of the users in the vicinity of a single base station. Values for the feedback gains $k_{i}$, the target SIRs $\gamma_{i}$, and the receiver noises $\nu_{i}$ are chosen in the region of those considered in [2], [15] so as 


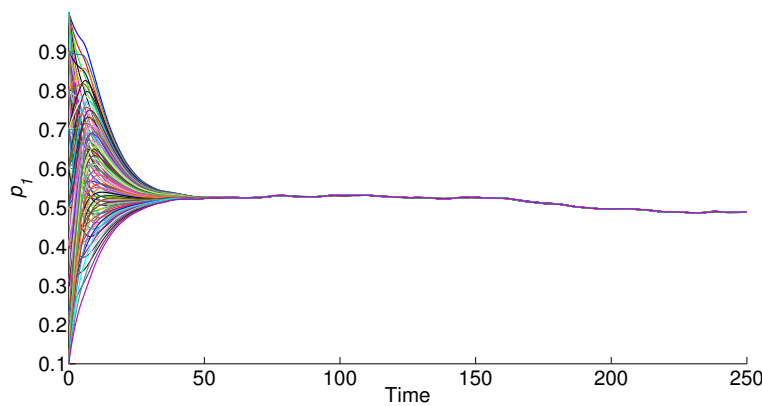

Fig. 3. The evolution of $p_{1}$ over time from 100 different initial conditions.

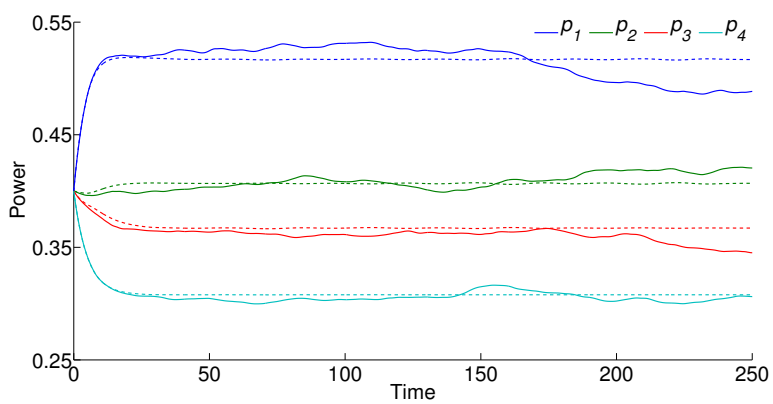

Fig. 4. Solid lines represent the power solutions for (14) with gains evolving according to (15). Dashed lines represent the power solutions for (14) with constant gains (18).

to give numerical simulations that are both physically relevant and insightful ${ }^{15}$. We fix the delays in the interval $[4,6]$. Using these values, the systems whose nonlinearities are $J^{\mathrm{min}}$ and $J^{\max }$ have respective equilibria

$$
q^{\min }=\left(\begin{array}{c}
0.2415 \\
0.2183 \\
0.2139 \\
0.2014
\end{array}\right) \text { and } q^{\max }=\left(\begin{array}{c}
3.3848 \\
2.3413 \\
1.2236 \\
0.7076
\end{array}\right),
$$

both of which are positive in all components. Therefore, (17) implies that (13) holds, and thus all of the assumptions of Proposition 1 are satisfied. Consequently, Proposition 1 gives a non-empty, positively invariant set, thereby guaranteeing the existence of trajectories along which the system nonlinearity (16), with arbitrary bounded delays, is bounded. This means that Theorem 3 is applicable here, meaning that all trajectories, independent of the initial conditions in the powers, must have the same asymptotic behavior.

In Fig. 2, we plot two-dimensional projections of various trajectories. Each plot is given twice ((a) pairs with (b), (c) with (d), and (e) with (f)), the first time with the initial conditions for the two components of the power vector not illustrated set to the constant value 0.4 (for all $t \in[-6,0]$ ) and the second time with them set to 2 . The initial values are indicated by open circles, and the projections of the trajectories themselves are the curves emanating from these data points. We observe that, in agreement with our above results, all the

\footnotetext{
${ }^{15}$ The scaling constant depends on the typical velocity and distance scales of the problem. In order to represent this setting, we have taken the values $0.5 \mathrm{~ms}^{-1}$ and $50 \mathrm{~m}$ respectively.
}

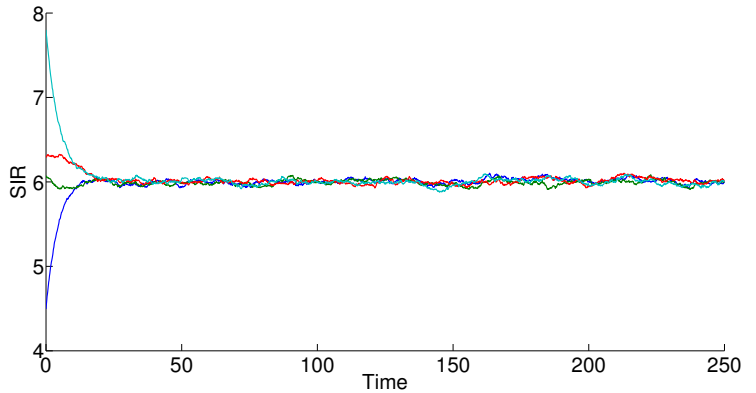

Fig. 5. Evolution of the four components of the signal-to-interference ratio. Here each target SIR $\gamma_{i}$ is 6 .

trajectories shown do appear to display the same asymptotic behavior. When we plot the evolution of any one particular component against time, as we do for $p_{1}$ in Fig. 3, we see clear evidence of this asymptotic convergence. The exact nature of the limiting behavior is clarified in Fig. 4, where we see that the asymptotic behavior of the power values for the present system (plotted with solid lines) appears to follow the general shape of the power value solutions for the system (14) with constant effective gains (18) (plotted with dashed lines), up to some random perturbative process.

Of particular significance to our understanding of the applicability of the algorithm in the present setting is the plot shown in Fig. 5 of the evolution of the signal-to-interference ratio for each node with time. As discussed in section III, in the time-independent case, the Foschini-Miljanic algorithm converges to a power distribution satisfying the given SIR target, provided that this is possible. We see here that the behavior when the link gains are time-varying is, in fact, similar in that our simulation shows each component of the SIR attaining oscillatory values close to the SIR target.

The foregoing simulation is for the particular values of the feedback gains $k_{i}$ and the delays stipulated previously, namely $k_{i}=0.2$ and delays fixed in the interval [4,6]. It follows, however, from Theorem 3 and the way that Proposition 1 has been applied in this section that the same asymptotic behavior should be observed irrespective of the values of these quantities. This is illustrated in Fig. 6, where we plot the component $p_{1}$ against time for several different solutions of (14) with gain evolution (15) in six distinct regimes. Again, it appears that all of the trajectories do indeed display the same asymptotic behavior in this component. Additionally, Fig. 6 makes clear the effect of these changes on the qualitative behavior of the system. Increasing the values of the $k_{i}$ corresponds to making the feedback within the system more aggressive. We observe from (b), (d), and (f) that the system then exhibits more rapid convergence of any two trajectories to one another, and also reacts more sharply to changes in the link gains, giving a more jagged response in the power values. Thus, larger values of these feedback gains are seen to give rise to more rapid convergence, allowing the system to better track time-variations in the system parameters, but will also yield more significant responses to stochastic fluctuations in measurements, potentially leading in 


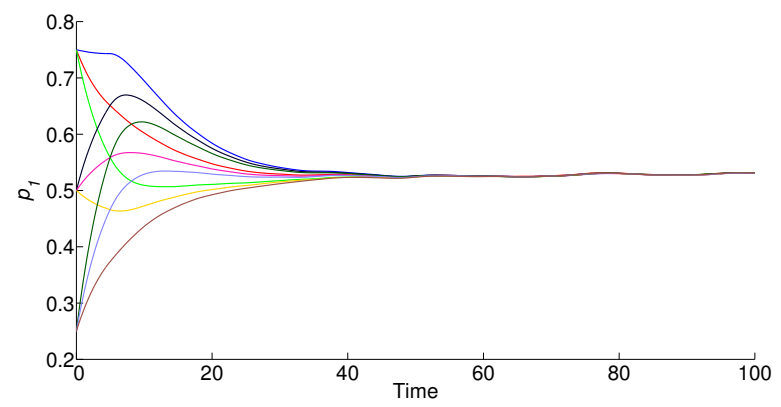

(a) $k_{i}=0.2$ with the original delays.

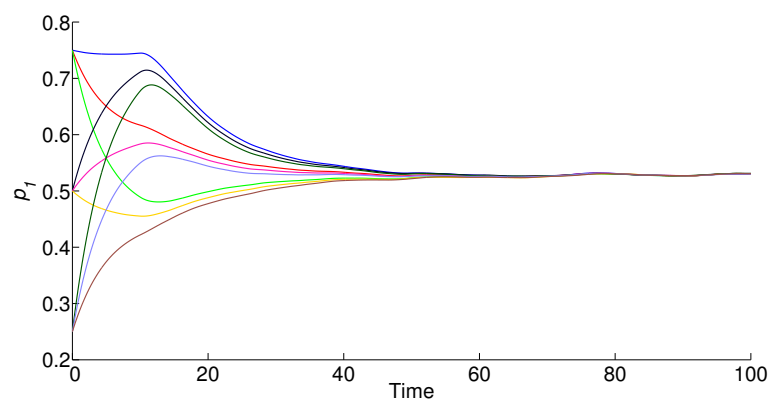

(c) $k_{i}=0.2$ with double the original delays.

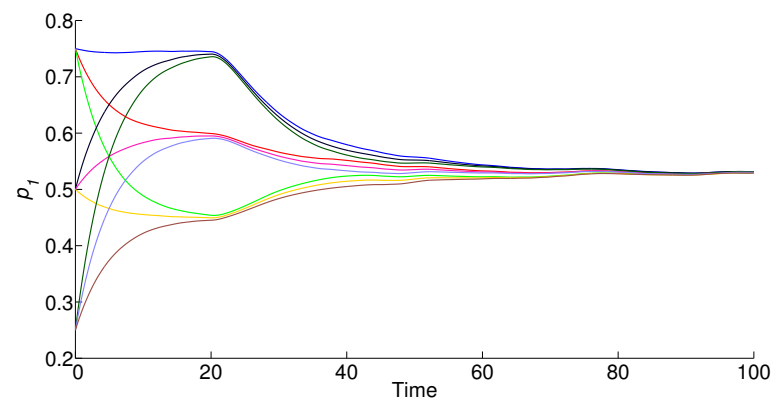

(e) $k_{i}=0.2$ with quadruple the original delays.

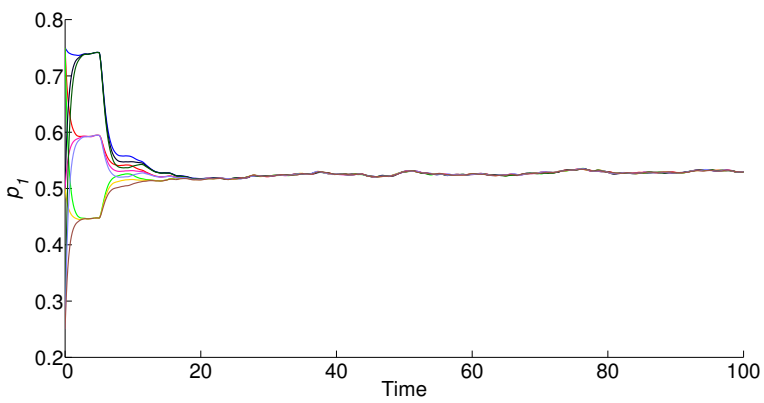

(b) $k_{i}=2$ with the original delays.

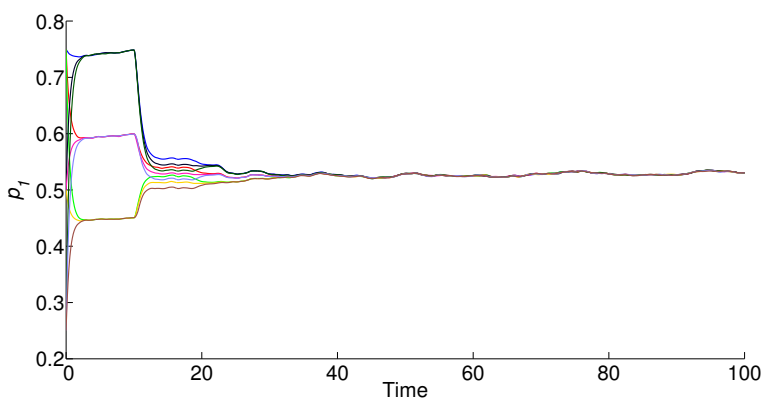

(d) $k_{i}=2$ with double the original delays.

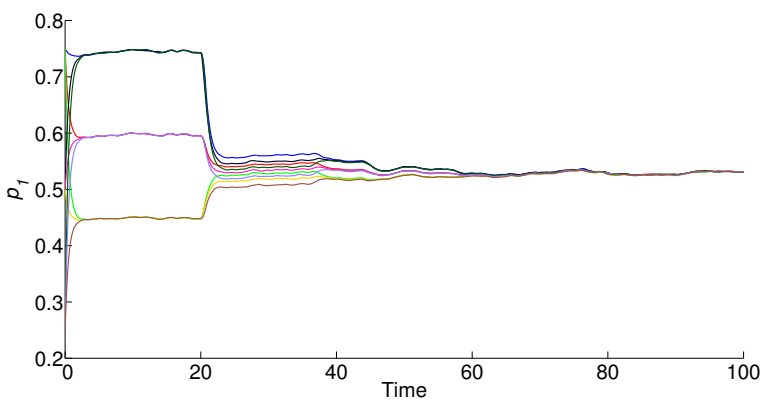

(f) $k_{i}=2$ with quadruple the original delays.

Fig. 6. Plots of $p_{1}$ against time for various solutions of system (14) for different values of the feedback gains and the delays.

practical applications to large transient deviations from the desired system performance. Therefore, appropriate feedback gain values must be chosen in order to ensure sufficiently rapid convergence while limiting the influence of stochastic disturbances. By contrast, the primary effect of increasing the delays is to increase the timescale of the initial transient behavior, leading to increased convergence time. Therefore, as expected, reductions in measurement and transmission delays are beneficial to the overall system performance.

\section{CONCLUSIONS}

We have considered a general class of algorithms for distributed power control in time-varying wireless networks. It has been shown that any bounded power distribution arising from these algorithms is uniformly asymptotically stable. Furthermore, even if the system includes heterogeneous, timevarying delays, any solution along which the nonlinearity is bounded must also be uniformly asymptotically stable. In both cases it was also shown that this stability is global, i.e. all trajectories have the same asymptotic behavior. These results greatly simplify the analysis of such systems, as they allow the long-term behavior of the wireless system to be determined even without knowledge of its initial state. In addition, the aforementioned convergence results have been exploited to derive time-invariant bounds on the asymptotic behavior of the trajectories when the system nonlinearities satisfy appropriate conditions. The Lyapunov approaches used within this context are also of independent interest as they could be of value in addressing other important open problems, such as those of guaranteeing stability in the presence of more involved dynamics and of quantifying the rate of convergence to the equilibrium trajectories.

\section{APPENDIX}

It should be noted that the results obtained in the main sections of this paper do not require the system feedback gains to be constant. In this appendix we illustrate this fact by extending the Lyapunov-Razumikhin approaches used in 
section IV to cover the case in which the static feedback gain factors $k_{I}$ are replaced by a more general class of time-varying passive, nonlinearities $g_{i}$ that satisfy a scalability condition. In particular, the system of interest ${ }^{16}$ is given by

$$
\frac{d p_{i}(t)}{d t}=g_{i}\left(t,-p_{i}(t)+I_{i}\left(t, p^{d_{i}}(t)\right)\right),
$$

where we stipulate that all of the functions $g_{i}: \overline{\mathbb{R}}_{+} \times \mathbb{R} \rightarrow \mathbb{R}$ must satisfy the following properties at all times $t$ :

i) $g_{i}(t, 0)=0$,

ii) $g_{i}(t, \alpha x) \leq \alpha g_{i}(t, x)$ for all $\alpha>1$ and all $x \in \mathbb{R}$,

iii) $g_{i}(t, x)-g_{i}(t, y) \geq z(x-y)$ for all $x>y$, where $z: \mathbb{R}_{+} \rightarrow \mathbb{R}_{+}$is a continuous, positive, non-decreasing function (independent of $i$ ).

Condition (ii) is a weak form of the scalability condition inherited from the interference nonlinearities $I_{i}$, while conditions (i) and (iii) are closely related to the conditions required for $g_{i}(t, x)$ to be an increasing passive function of $x$.

Theorem 4: Suppose that $p=P(t)$ is a solution of equation (19) satisfying $P(\theta) \geq 0$ for all $\theta \in[-r, 0]$. Suppose further that there exists a positive constant $C$ such that $I_{i}\left(t, P^{d_{i}}(t)\right)<C$ for all $i \in\{1,2, \ldots, N\}$ and all $t \geq 0$. Then any trajectory $p(t)$ with $p(\theta) \geq 0$ for $\theta \in[-r, 0]$ will tend uniformly to $P(t)$ as $t \rightarrow \infty$.

Proof: Just as in the proof of Theorem 3, we can convert a trajectory with initial condition merely non-negative to one with initial condition strictly positive by means of a shift of the time-origin. Therefore, we need consider only trajectories with strictly positive initial condition in what follows.

In order to investigate these systems, we again introduce the change-of-variables (4), yielding the equations

$$
\begin{array}{r}
\frac{d \pi_{i}}{d t}=\frac{1}{P_{i} e^{\pi_{i}}}\left[g_{i}\left(t,-P_{i} e^{\pi_{i}}+I_{i}\left(t, \operatorname{diag}\left(e^{\pi_{j}^{d_{i}}}\right) P^{d_{i}}\right)\right)\right. \\
\left.-e^{\pi_{i}} g_{i}\left(t,-P_{i}+I_{i}\left(t, P^{d_{i}}\right)\right)\right] .
\end{array}
$$

The legitimacy of this transformation follows from Lemma 3 as previously, invoking the fact that $g(t, x)>0$ for all $x>0$. Similarly, the fact that $g(t, x)<0$ for all $x<0$ means that the nonlinearity bound $I_{i}\left(t, P^{d_{i}}\right)<C$ implies an upper bound $B$ on all components of the trajectory $p=P(t)$.

Let us now introduce the same candidate LyapunovRazumikhin function as used in the proof of Theorem 3, $V(\pi)=\max _{i}\left|\pi_{i}\right|=\left|\pi_{i_{m}}\right|$. We now suppose that the condition (9) holds, whence

$$
q\left(\left|\pi_{i_{m}(t)}(t)\right|\right) \geq\left|\pi_{j}^{d_{i_{m}(t)}}\right|
$$

for some function $q$ which we shall show can be chosen to satisfy the conditions required in Theorem 1 . In order to do this, we shall let $q(s)=s+f(s)$ for some $f$ assumed nonnegative. Then, writing $i$ for $i_{m}$, there are again two significant cases:

i) $\pi_{i}>0$, in which case (21) gives $\pi_{j}^{d_{i}}-q\left(\pi_{i}\right) \leq 0$ and we get from case (i) in the proof of Theorem 3 that

\footnotetext{
${ }^{16}$ We present here only the argument for the delayed case; however, in the absence of delays, it can readily be seen that analogous modifications of the arguments of Theorem 2 will extend this result to this more general situation as well.
}

if $f \leq f_{1}$ for some continuous function $f_{1}$ satisfying $f_{1}(0)=0$ and $f_{1}(s)>0$ for $s>0$, then if

$$
1<e^{f_{1}\left(\pi_{i}\right)}<1+\frac{\delta\left(e^{\pi_{i}}\right)}{2 C}
$$

we must have

$$
e^{-\pi_{i}} I_{i}\left(t, \operatorname{diag}\left(e^{\pi_{j}^{d_{i}}}\right) P^{d_{i}}\right)-I_{i}\left(t, P^{d_{i}}\right)<-\frac{\delta\left(e^{\pi_{i}}\right)}{2} .
$$

Now, (20) gives

$$
\begin{array}{r}
\dot{\pi}_{i}=\frac{1}{P_{i}}\left[e^{-\pi_{i}} g_{i}\left(t,-P_{i} e^{\pi_{i}}+I_{i}\left(t, \operatorname{diag}\left(e^{\pi_{j}^{d_{i}}}\right) P^{d_{i}}\right)\right)\right. \\
\left.-g_{i}\left(t,-P_{i}+I_{i}\left(t, P^{d_{i}}\right)\right)\right] \\
\leq \frac{1}{P_{i}}\left[g_{i}\left(t,-P_{i}+e^{-\pi_{i}} I_{i}\left(t, \operatorname{diag}\left(e^{\pi_{j}^{d_{i}}}\right) P^{d_{i}}\right)\right)\right. \\
\left.-g_{i}\left(t,-P_{i}+I_{i}\left(t, P^{d_{i}}\right)\right)\right],
\end{array}
$$

by property (ii). But now (22) implies in particular that

$$
-P_{i}+e^{-\pi_{i}} I_{i}\left(t, \operatorname{diag}\left(e^{\pi_{j}^{d_{i}}}\right) P^{d_{i}}\right)<-P_{i}+I_{i}\left(t, P^{d_{i}}\right),
$$

whence property (iii) applies to (23) to yield

$$
\begin{aligned}
\dot{\pi}_{i} & \leq-\frac{1}{P_{i}} z\left(I_{i}\left(t, P^{d_{i}}\right)-e^{-\pi_{i}} I_{i}\left(t, \operatorname{diag}\left(e^{\pi_{j}^{d_{i}}}\right) P^{d_{i}}\right)\right) \\
& \leq-\frac{1}{B} z\left(\frac{\delta\left(e^{\pi_{i}}\right)}{2}\right),
\end{aligned}
$$

invoking the inequality (22) and the upper bound on $P_{i}(t)$. We note finally that $z\left(\frac{\delta\left(e^{\pi_{i}}\right)}{2}\right)$ is a continuous, strictly positive, non-decreasing function of $\pi_{i}$, with $\lim _{\pi_{i} \downarrow 0} z\left(\frac{\delta\left(e^{\pi_{i}}\right)}{2}\right)=0$.

ii) $\pi_{i}<0$, in which case (21) gives $\pi_{j}^{d_{i}}+q\left(-\pi_{i}\right) \geq 0$ and then case (ii) in the proof of Theorem 3 gives

$$
e^{-\pi_{i}} I_{i}\left(t, \operatorname{diag}\left(e^{\pi_{j}^{d_{i}}}\right) P^{d_{i}}\right)-I_{i}\left(t, P^{d_{i}}\right)>\frac{\delta\left(e^{-\pi_{i}}\right)}{2}
$$

when $f \leq f_{2}$ for some continuous function $f_{2}$ satisfying $f_{2}(0)=0$ and $f_{2}(s)>0$ for $s>0$ and

$$
1-\frac{\delta\left(e^{-\pi_{i}}\right)}{2 C}<e^{-f_{2}\left(-\pi_{i}\right)}<1 .
$$

In this case, the application of property (ii) to (20) gives

$$
\begin{array}{r}
\dot{\pi}_{i}=\frac{1}{P_{i}}\left[e^{-\pi_{i}} g_{i}\left(t,-P_{i} e^{\pi_{i}}+I_{i}\left(t, \operatorname{diag}\left(e^{\pi_{j}^{d_{i}}}\right) P^{d_{i}}\right)\right)\right. \\
\left.-g_{i}\left(t,-P_{i}+I_{i}\left(t, P^{d_{i}}\right)\right)\right] \\
\geq \frac{1}{P_{i}}\left[g_{i}\left(t,-P_{i}+e^{-\pi_{i}} I_{i}\left(t, \operatorname{diag}\left(e^{\pi_{j}^{d_{i}}}\right) P^{d_{i}}\right)\right)\right. \\
\left.-g_{i}\left(t,-P_{i}+I_{i}\left(t, P^{d_{i}}\right)\right)\right] .
\end{array}
$$

Then invoking (24) together with property (iii) analogously to case (i), we obtain

$$
\begin{aligned}
\dot{\pi}_{i} & \geq \frac{1}{P_{i}} z\left(e^{-\pi_{i}} I_{i}\left(t, \operatorname{diag}\left(e^{\pi_{j}^{d_{i}}}\right) P^{d_{i}}\right)-I_{i}\left(t, P^{d_{i}}\right)\right) \\
& \geq \frac{1}{B} z\left(\frac{\delta\left(e^{\pi_{i}}\right)}{2}\right) .
\end{aligned}
$$




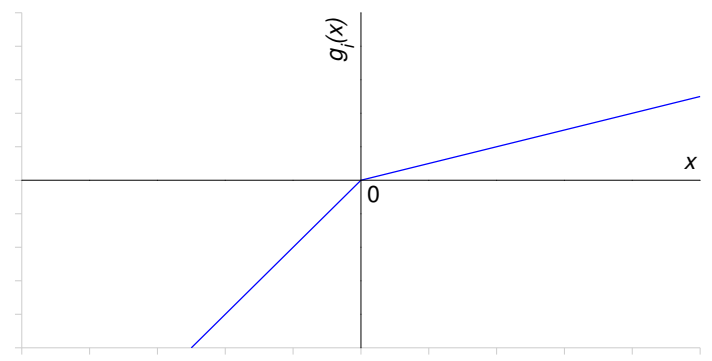

Fig. 7. An example of a feedback gain profile permitted by the extension discussed in this appendix.

Finally, we note again that this is a continuous, strictly positive, non-decreasing function of $-\pi_{i}$, with $\lim _{\pi_{i} \uparrow 0} z\left(\frac{\delta\left(e^{-\pi_{i}}\right)}{2}\right)=0$.

To complete our proof of the result, let us define $f(s)=$ $\min \left\{f_{1}(s), f_{2}(s)\right\}$ and $w(s)=\frac{1}{B} z\left(\frac{\delta\left(e^{s}\right)}{2}\right)$ for all $s \geq 0$. With these definitions, if $q(s)=s+f(s)$, we have $q$ and $w$ both continuous and non-decreasing, and $q(s)>s, w(s)>0$ for all $s>0$. Moreover, whenever (9) holds, $\dot{V}(\pi) \leq-w\left(\left|\pi_{i_{m}}\right|\right)$, whence all of the conditions of Theorem 1 are satisfied.

Finally, noting the radial unboundedness of the LyapunovRazumikhin function $V(\pi)=\max _{i}\left|\pi_{i}\right|$ and recalling our observation at the beginning of the proof about how to deal with trajectories with initial conditions possibly equal to zero completes the proof of the result.

Remark 4: Using the fact that property (iii) means that each $g_{i}(t, x)$ must be strictly increasing in $x$, it can easily be shown that Proposition 1 also holds for the generalized system (19).

The extension described in the foregoing discussion permits the use of modified feedback gain profiles, which may be of practical benefit in the application of these power control schemes. One such example is illustrated in Fig. 7; this entails the use of a greater feedback gain factor $k_{i}$ in the negative region than in the positive region, thereby endowing the system (19) with a more aggressive decrease rule than increase rule. Such an aggressive decrease rule allows for rapid power decrease when the signal power is much greater than the interference value, which can be of significant benefit in situations in which the consideration of users' battery life is important.

\section{REFERENCES}

[1] J. Zander, "Distributed co-channel interference control in cellular radio systems," IEEE Transactions on Vehicular Technology, vol. 41, no. 3, pp. 305-311, Aug. 1992.

[2] G. Foschini and Z. Miljanic, "A simple distributed autonomous power control algorithm and its convergence," IEEE Transactions on Vehicular Technology, vol. 43, no. 4, pp. 641-646, Nov. 1993

[3] M. Bambos, S. C. Chen, and G. Pottie, "Radio link admission algorithms for wireless networks with power control and active link quality protection," IEEE INFOCOM, pp. 97-104, Apr. 1995.

[4] R. D. Yates, "A framework for uplink power control in cellular radio systems," IEEE Journal on Selected Areas in Communications, vol. 49, no. 7, pp. 1341-1347, Sep. 1995.

[5] M. Xiao, N. B. Shroff, and E. K. P. Chong, "A utility-based power control scheme in wireless cellular systems," IEEE/ACM Transactions on Networking, vol. 11, no. 2, pp. 210-221, Apr. 2003.
[6] T. ElBatt and A. Ephremides, "Joint scheduling and power control for wireless ad hoc networks," IEEE Transactions on Wireless Communications, vol. 3, no. 1, pp. 74-85, Jan. 2004.

[7] N. Stefanovic and L. Pavel, "A stability analysis with time-delay of primal dual power control in optical links," Automatica, vol. 45, no. 5, pp. 1319-1325, May 2009.

[8] A. Möller and U. T. Jönsson, "Stability of high order distributed power control," Proceedings of the 48th IEEE Conference on Decision and Control, pp. 4963-4970, Dec. 2009.

[9] E. Devane and I. Lestas, "Stability of a general class of distributed algorithms for power control in time-dependent wireless networks,", Proceedings of the 51st IEEE Conference on Decision and Control, pp. 3922-3927, Dec. 2012.

[10] R. D. Yates and C. Y. Huang, "Integrated power control and base station assignment," IEEE Transactions on Vehicular Technology, vol. 44, no. 3 , pp. 638-644, Aug. 1995.

[11] S. V. Hanly, "An algorithm for combined cell-site selection and power control to maximize cellular spread spectrum capacity," IEEE Journal on Selected Areas in Communications, vol. 13, no. 7, pp. 1332-1340, Sep. 1995

[12] S. V. Hanly, "Capacity and power control in spread spectrum macrodiversity radio networks," IEEE Transactions on Communications, vol. 44, no. 2, pp. 247-256, Feb. 1996.

[13] C. Sung and K. Leung, "A generalized framework for distributed power control in wireless networks," IEEE Transactions on Information Theory, vol. 51, no. 7, pp. 2625-2635, Jul. 2005.

[14] M. Schubert and H. Boche, "A generic approach to QoS-based transceiver optimization," IEEE Transaction on Communications, vol. 55, no. 8, pp. 1557-1566, Aug. 2007.

[15] I. Lestas, "Distributed power control in wireless networks: stability and delay independence," Proceedings of the 48th IEEE Conference on Decision and Control, pp. 7074-7079, Dec. 2009.

[16] H. K. Khalil, Nonlinear Systems. Prentice Hall, 1996.

[17] N. P. Bhatia and G. P. Szego, Stability Theory of Dynamical Systems. Springer-Verlag Berlin Heidelberg, 2002.

[18] J. K. Hale and S. M. Lunel, Introduction to Functional Differential Equations. Springer-Verlag New York, 1993.

[19] B. S. Razumikhin, "On the stability of systems with a delay," (in Russian) Prikladnava Matematika i Mekhanika, vol. 20, pp. 500-512, 1956.

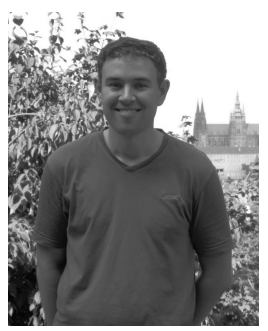

Eoin Devane received the M.Math. degree (firstclass) in mathematics from University of Oxford (Merton College) in 2011. Since October 2011, he has been a Ph.D. student with the Cambridge Centre for Analysis, Faculty of Mathematics, University of Cambridge (Pembroke College), supported by an EPSRC doctoral training grant.

His research interests include network optimization and control, with applications to communication networks and electrical power systems.

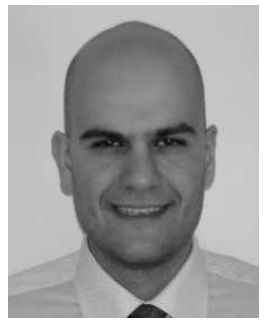

Ioannis Lestas received the B.A. (Starred First) and M.Eng. (Distinction) degrees in Electrical Engineering and Information Sciences and the Ph.D. degree in control theory from the University of Cambridge (Trinity College) in 2002 and 2007, respectively. His doctoral work was performed as a Gates Scholar and Trinity College Research Scholar. In 2006 he was elected to a Junior Research Fellow of Clare College, University of Cambridge and he was awarded a five year Royal Academy of Engineering research fellowship in 2008. He is currently a University Lecturer at Cambridge, Department of Engineering and a fellow of Clare College.

His research interests include analysis and control of large scale networks focusing on issues of scalability, robustness and fundamental limitations with applications in power systems, data networks, multiagent systems and biological networks. 